\title{
Quantitative-Genetic Models and Changing Environments
}

Reinhard Bürger (reinhard.buerger@univie.ac.at)

Christoph Krall (christoph.krall@univie.ac.at)

\section{Approved by}

Ulf Dieckmann (dieckman@iiasa.ac.at)

Program Leader, Adaptive Dynamics Network

November 2004 


\section{Contents}

1 Introduction 1

2 Quantitative Genetics and Response to Selection 2

3 Adaptation and Extinction in Changing Environments $\quad 7$

Sustained directional change . . . . . . . . . . . . . . . . . . . 7

Pleiotropy and changing optima $\ldots \ldots \ldots \ldots$. . . . . . . . . . . 10

Periodic change . . . . . . . . . . . . . . . . . . . . . 11

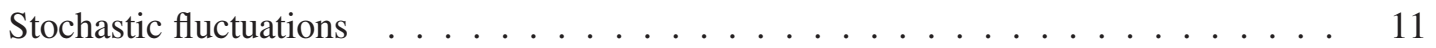

Single abrupt change . . . . . . . . . . . . . . . . . . 13

4 Concluding Comments $\quad 13$ 


\author{
About the Authors \\ Reinhard Bürger \\ Department of Mathematics \\ University of Vienna \\ Strudlhofgasse 4, A-1090 Vienna, Austria \\ Christoph Krall \\ Department of Mathematics \\ University of Vienna \\ Strudlhofgasse 4, A-1090 Vienna, Austria
}

\title{
Acknowledgements
}

This work was financially supported by the Austrian Science Foundation (FWF),

Projects P10689-MAT and P12865-MAT. 


\title{
Quantitative-Genetic Models and Changing Environments
}

\author{
Reinhard Bürger \\ Christoph Krall
}

\section{Introduction}

Mutation is the ultimate source of genetic variability. However, a large fraction of mutations reduce the fitness of the individuals in which they occur (Chapter 7 in Ferrière et al. 2004). The evolutionary consequences of mutations with an unconditionally deleterious effect are manifold and have been the subject of intense investigation (Charlesworth and Charlesworth 1998; Chapter 9 in Ferrière et al. 2004). Since many mutations affect several traits and the developmental pathways are complex, their fitness effects may also depend on the genetic background in which they occur, and on the kind of selective pressure to which the population is exposed. For instance, if for a given trait, say birth weight, there is an optimal phenotype, a mutation that increases birth weight will be detrimental if it arises in a genotype that, otherwise, would have the optimal or a higher birth weight, but will be beneficial in other genotypes. In a changing environment, the selective value of an allele will change with time if different values of a trait affected by the allele are favored at different times. The fitness effect of a given mutation, therefore, depends on the effect it has on this trait, and on the current environment. Hence, a newly introduced allele may, in spite of its immediate adversary effect on fitness, prove to be beneficial at a later stage of the population history. Mutation itself thereby gains an additional role as a provider of the genetic variation that allows adaptation to occur. In this chapter, we evaluate the role of genetic variation, as caused by mutation, for population persistence if environmental change induces selection on one or more quantitative traits.

For the present purpose, environmental changes may be grouped roughly according to the mode in which they occur in time:

- Stochastic fluctuations of a certain parameter around a constant mean (e.g., temperature in tropical regions);

- Periodic fluctuations around a constant mean (e.g., seasonal fluctuations, oscillations in the life cycles of prey, predators, or parasites) that are at least partially predictable;

- Directional changes, such as global climatic changes, increasing concentration of certain substances because of increasing pollution, or gradual loss of habitat through human settlement or spread of a predator or pest (see Chapter 5 in Ferrière et al. 2004);

- Single abrupt changes in the local environment, as caused by the sudden introduction of pests or pesticides; an abrupt change may also be faced by a founding colony in a novel habitat.

The different types of changes can be superimposed in arbitrary combination, and thereby pose different challenges upon the population and prompt different responses. These may range from immediate extinction to evolution sustained over long periods, possibly resulting in speciation (Chapter 7 in Ferrière et al. 2004). 
The response to environmental change will be influenced in various ways by ecological, demographic, and genetic factors. Ecological considerations take into account that environmental changes often influence a population not only by direct effects, but also via their effects on the focal population's preys, predators, mutualistic partners, or competitors. The interplay between direct and indirect effects determines the selective forces. So what may be experienced as a changing environment for a single species may, at a higher level of observation, be described as the intrinsic dynamics of the ecosystem under constant environmental conditions (see Chapters 16 and 17 in Ferrière et al. 2004).

Demographic factors include the reproductive system, population size, intrinsic population growth rate, migration patterns, and so on. For instance, small populations are more affected by stochastic influences, and a high growth rate enhances population recovery after a bottleneck caused by a catastrophic event (see Chapters 2 to 4 in Ferrière et al. 2004).

Consideration of the relevant properties of the genetic system of the population must include at least the degree of ploidy, the number of loci, and the way these interact, and must determine the phenotype (e.g., pleiotropy, if one gene has effects on several traits), recombination, and mutation. Mutation is crucial in the long run because it generates new genetic variability. Recombination breaks associations between alleles at different loci, and thus allows beneficial alleles to spread through the population and accelerates the elimination of deleterious alleles. Pleiotropy may impede adaptation by coupling the selectively advantageous change of one character with the maladaptation of pleiotropically connected characters.

The combined action of all these factors mentioned ultimately determines the genetic composition of the population and, via the amount of genetic variation, the rate of response to environmental challenges. For various scenarios of environmental change, we elucidate the:

- Role of genetic variation for adaptation;

- Rate of adaptive response; and

- Extinction risk of a population, as measured by the expected extinction time.

Demographic and genetic parameters are incorporated explicitly into the models, whereas ecological interactions are subsumed in the parameters that describe environmental change and selection.

\section{Quantitative Genetics and Response to Selection}

Quantitative characters are traits that exhibit continuous or almost continuous variation and can be measured on a metric scale. Typical examples are weight, height, various morphological measurements, yield, or fitness. Usually, such traits are controlled by a large number of gene loci, often with small effects. Since individuals in a population differ in their trait values, the state of a population is best described by the probability distribution of the trait. Quite often, the mean value and the variance are sufficient to predict the evolutionary response of a population to selection. In contrast to the frequencies of the genes that determine the character, the values of the mean, the variance, and (sometimes) the higher moments can be estimated accurately from real data.

Pioneering analyses to elucidate the genetic basis of inheritance and the response to selection of the mean of a quantitative character were made by Galton (1889), Pearson (1903), Fisher (1918), and Wright (1921), and their students Smith (1936) and Lush (1937). While the analyses of Galton (1889) and Pearson (1903) were of a purely statistical nature and based on regression theory, Fisher reconciled their biometric description with Mendelian genetics by assuming that a large number of unlinked loci with small additive effects determine the character. The work of Fisher (1918) and Wright (1921) forms the basis of classic quantitative genetic theory and its applications to animal and plant breeding (see, e.g., Bulmer 1980; Mayo 1987; Falconer and Mackay 1996; 
Box 1 The classic additive genetic model

Quantitative traits are influenced by genes at many $(\ell)$ loci and by the environment. Genetically identical individuals may have different phenotypes because of external conditions (e.g., nutrition), developmental "noise", and cytoplasmatic effects. All these are lumped together into the so-called environmental contribution $E$, which (in the simple model discussed here) is assumed to be independent of the genotype and normally distributed with a mean of zero and a variance of $V_{E}$. Alleles at each locus have an effect on the character, measured by a real number. Let $\bar{x}_{i}$ and $x_{i}$ denote the allelic effect of the maternally and paternally inherited alleles, respectively, at locus $i$. The fundamental assumption is that the alleles between the loci interact additively and, in the diploid case (as assumed here), show no dominance. Thus, the phenotypic value $P$ of an individual is assumed to be

$$
P=G+E=\sum_{i=1}^{\ell}\left(\bar{x}_{i}+x_{i}\right)+E,
$$

where $G$ is the genotypic value. Since the environmental contribution is scaled to have zero mean, the mean phenotypic value $\bar{P}$ equals the mean genotypic value $\bar{G}$. A consequence of the assumption of no genotype-environment interaction is that the mean phenotypic variance $V_{P}$ can be decomposed into

$$
V_{P}=V_{G}+V_{E}
$$

where $V_{G}$ is the (additive) genetic variance (i.e., the variance of genotypic values). The assumption of additivity of allelic effects rests on the fundamental concept of the average or additive effect (Fisher 1930, 1941) and may be viewed as a least-squares approximation. Thus, the additive effects are found by an analysis of variance; indeed, Fisher invented the analysis of variance for this purpose. Often, an appropriate scale of measurement can be chosen so that the additivity assumption is a close approximation (Falconer and Mackay 1996). The variance of additive effects, in this case equal to $V_{G}$, is the main determinant of the response to selection.

Let $S$ denote the selection differential, that is the within-generation difference between the mean phenotypes $\bar{P}$ (before selection) and $\bar{P}_{S}$ (after selection but before reproduction). The expected change in the mean phenotype across generations is then equal to

$$
\Delta \bar{P}=h^{2} S,
$$

where $h^{2}=V_{G} / V_{P}$, the ratio of additive genetic to phenotypic variance, and is called the heritability. It measures the fraction of variance that is heritable. Equation (c) is called the breeder's equation and is of fundamental importance because it allows prediction of the expected selection response from measurable quantities (see Mayo 1987; Falconer and Mackay 1996).

Lynch and Walsh 1998; Bürger 2000). Box 1 summarizes the basic aspects of the additive model of quantitative genetics.

The so-called breeder's equation, Equation (c) in Box 1, allows prediction of the change between generations on the basis of the selection differential. For many questions of evolutionary interest, however, selection is conveniently modeled by a fitness function $W(P)$, which assigns a fitness value to each phenotypic value $P$. In the simplest case, it measures the probability that an individual survives viability selection. Lande (1976, 1979, and later articles) extended the classic approach and derived dynamic equations for the change of mean phenotype of a set of quantitative characters in terms of the additive genetic covariance matrix and the so-called selection gradient. The fundamentals of his theory are summarized in Box 2 and form the basis for the subsequent analysis herein.

We now set up the general model on which the present results are based. A finite, sexually reproducing population of diploid individuals is assumed; it mates at random and, with respect to the traits considered, it has equivalent sexes. For simplicity, fitness is determined by a single quantitative character under Gaussian stabilizing selection on viability, with the optimum phenotype $\hat{P}_{t}$ 
Box 2 Lande's phenotypic model of selection

Consider a randomly mating, large population such that random genetic drift can be ignored and assume that the sexes are equivalent with respect to the characters considered. The phenotype $P$ of an individual is characterized by measurements of $n$ traits, that is $P=\left(P_{1}, \ldots, P_{n}\right)^{T}$, where ${ }^{T}$ denotes vector transposition. In analogy with the theory in Box 1 , a decomposition $P=G+E$ is assumed with independent distributions of $G$ and $E$ that are multivariate normal with mean vectors $\bar{G}$ and 0 , and covariance matrices $V_{G}$ and $V_{E}$. Thus, the phenotypic covariance matrix is $V_{P}=$ $V_{G}+V_{E}$. The normality assumption is justified if, as is often the case in practice, a scale can be found on which phenotypic values are approximately normally distributed (Falconer and Mackay 1996). This is also expected from the central limit theorem, because many loci, as well as environmental effects, contribute to quantitative traits.

If $W(P)$ denotes the fitness of an individual with phenotype $P$, then the mean fitness of the population is

$$
\bar{W}=\int \phi(P) W(P) d P,
$$

where $\phi(P)$ is the (multivariate) probability density of $P$. After selection, the mean vector is

$$
\bar{P}_{S}=\frac{1}{\bar{W}} \int P \phi(P) W(P) d P,
$$

so that the vector of selection differentials is $S=\bar{P}_{S}-\bar{P}$. In a generalization of Equation (c) in Box 1, the change between generations is calculated to be

$$
\Delta \bar{P}=V_{G} V_{P}^{-1} S .
$$

Let $\nabla$ denote the gradient operator, that is $\nabla g=\left(\partial g / \partial x_{1}, \ldots, \partial g / \partial x_{n}\right)^{T}$ is the vector of partial derivatives of the real-valued function $g$ depending on the $n$ arguments $x_{1}, \ldots, x_{n}$. Then, the mean fitness, expressed as a function of the vector of mean phenotypes $\bar{W}\left(\bar{P}_{1}, \ldots, \bar{P}_{n}\right)$, can be viewed as an adaptive topography that determines the response of the mean phenotype to selection. Indeed, Lande $(1976,1979)$ derived the fundamental relation

$$
\Delta \bar{P}=V_{G} \nabla \ln \bar{W},
$$

where the derivatives in the selection gradient $\nabla \ln \bar{W}$ are taken with respect to $\bar{P}_{1}, \ldots, \bar{P}_{n}$. For a single trait and with the notation from Box 1, Equation (c) reduces to

$$
\Delta \bar{P}=V_{G} \frac{1}{\bar{W}} \frac{d \bar{W}}{d \bar{P}} .
$$

Lande and others have applied this theory (and several generalizations) to numerous problems of evolutionary biology. The application to long-term evolution, however, requires knowledge of the genetic variances and covariances. Many of these analyses relied on the assumption that the phenotypic and genetic covariance matrices change on a much slower time scale than the mean values. These assumptions have been the subject of intense discussion and analysis (see Turelli 1984, 1988; Barton and Turelli 1989; Turelli and Barton 1994; Bürger 2000).

exhibiting temporal change (but see subsection Pleiotropy and changing optima in Section 3 for some multivariate results). Thus, the more a phenotype deviates from the optimum the lower is its fitness. In mathematical terms, the fitness (viability) of an individual with phenotypic value $P$ is conveniently described as

$$
W(P, t)=\exp \left(-\frac{\left(P-\hat{P}_{t}\right)^{2}}{2 \omega^{2}}\right),
$$

where $\omega^{2}$ is inversely proportional to the strength of stabilizing selection and independent of the generation number $t$. Selection acts only through viability selection, and each individual produces 
$b$ offspring. Initial populations are assumed to be in a stationary state with respect to stabilizing selection and genetic mechanisms when environmental change commences.

The following types of environmental change are modeled here:

- A phenotypic optimum that moves at a constant rate $\kappa$ per generation,

$$
\hat{P}_{t}=\kappa t
$$

- A periodically fluctuating optimum,

$$
\hat{P}_{t}=A \sin (2 \pi t / T),
$$

where $A$ and $T$ measure amplitude and period of the fluctuations, respectively;

- An optimum fluctuating randomly about its average position;

- A single abrupt shift of the optimum phenotype.

Under each of these models, the population experiences a mixture of directional and stabilizing selection. Such models of selection have been investigated previously by Lynch et al. (1991), Charlesworth (1993a, 1993b), Lynch and Lande (1993), Bürger and Lynch (1995, 1997), Kondrashov and Yampolsky (1996a, 1996b), Lande and Shannon (1996), Bürger (1999), and Bürger and Gimelfarb (2002).

The quantitative character under consideration is assumed to be determined by $n$ mutationally equivalent, recombining loci. The additive genetic model of Box 1 is assumed and, as usual, the scale of measurement is normalized such that $V_{E}=1$. Neither the genetic nor the phenotypic variance is assumed to be constant, which is indicated by a subscript $t$. The parameter $V_{S}=$ $\omega^{2}+V_{E}=\omega^{2}+1$ is used to describe the strength of stabilizing selection on the genotypic values $G$ (Lande 1975). We then have $V_{S}+V_{G}=\omega^{2}+V_{P}$.

Since this chapter is concerned with finite populations of effective size $N_{e}$, theoretical predictions are needed for the distribution of the mean phenotype, because it will fluctuate around its deterministically expected position. Let

$$
s_{t}=\frac{V_{G, t}}{V_{G, t}+V_{S}}
$$

be a measure for the strength of selection. Under the assumption of a Gaussian distribution of phenotypic values and a constant genetic variance, the distribution of the mean phenotype $\bar{P}_{t+1}$ in generation $t+1$, conditional on $\bar{P}_{t}$ and $\hat{P}_{t}$, is Gaussian. Its expectation is given by

$$
\mathbb{E}\left(\bar{P}_{t+1} \mid \bar{P}_{t}, \hat{P}_{t}\right)=\bar{P}_{t}+s_{t}\left(\hat{P}_{t}-\bar{P}_{t}\right) .
$$

This is a consequence of Equation (d) in Box 2 because mean fitness is calculated to be

$$
\bar{W}_{t}=\frac{\omega}{v_{t}} \exp \left(-\frac{1}{2}\left(\bar{P}_{t}-\hat{P}_{t}\right)^{2} / v_{t}^{2}\right)
$$

where $v_{t}^{2} \approx V_{S}\left(1+1 / 2 N_{e}\right)+V_{G, t}$ (Latter 1970; Lande 1976; Bürger and Lynch 1995). These equations are very general and hold for arbitrary fitness functions of the form in Equation (1), so long as the phenotypic values remain approximately Gaussian.

Under prolonged environmental change, mean fitness may become very low. Since it is assumed that individuals can only produce a finite number $b$ of offspring, a constant population size cannot necessarily be maintained because the (multiplicative) growth rate

$$
R_{t}=b \bar{W}_{t}
$$

may fall below 1 . Therefore, a simple kind of density-dependent population regulation is imposed to ensure that the population size is close to the carrying capacity $K$, as long as the growth rate $R_{t}$ is larger than 1, but allows extinction otherwise (Box 3). 
Box 3 A numerical model for adaptation in changing environments

The simulation model has been adapted from that used in Bürger et al. (1989) and Bürger and Lynch (1995). It uses direct Monte Carlo simulation to represent each individual and each gene. The genotypic value of the character is determined by $\ell$ additive loci with no dominance or epistasis. In the present investigation $\ell=50$ was chosen.

Following Crow and Kimura's (1964) continuum of alleles model, at each locus an effectively continuous distribution of possible effects for mutants is assumed. Thus, provided an allele with effect $x$ gives rise to a mutation, the effect of the mutant is $x+\xi$, where $\xi$ is drawn from a distribution with mean zero, variance $\gamma^{2}$, and no skewness. Hence, the number of possible segregating alleles per locus is limited only by population size. The mutation rate per haploid locus is denoted by $u$, the genomic mutation rate by $U$, and the variance introduced by the mutation per generation per zygote by $V_{m}=U \gamma^{2}$. Unless otherwise stated, a Gaussian distribution of mutational effects with a mean of zero and variance $\gamma^{2}=0.05$ and a (diploid) genomic mutation rate of $U=0.02$ per individual and generation are assumed. This implies that the variance introduced through mutation in each generation is $V_{m}=0.001$. These values have been suggested as gross averages by reviews of empirical data (see Lande 1975; Turelli 1984; Lynch and Walsh 1998).

The phenotypic value of an individual is obtained from the genotypic value by adding a random number drawn from a normal distribution with a mean of zero and variance $V_{E}=1$.

The generations are discrete, and the life cycle consists of three stages:

- Random sampling without replacement of a maximum of $K$ reproducing adults from the surviving offspring of the preceding generation;

- Production of offspring, including mutation, segregation, and recombination;

- Viability selection according to Equation (1).

Modification of this model to allow for two pleiotropically related traits is straightforward. Each allele is now written as a vector $(x, y)$, in which the two entries represent the effect of the allele on each of the traits it influences. Mutation is modeled by the addition of a vector $(\xi, \zeta)$, the components being drawn independently from a Gaussian distribution as in the single-character case. Viability selection acts on both characters and is modeled by a bivariate extension of the fitness function given by Equation (1). It is assumed that the optimum moves in the direction of the first trait, thus leading to directional selection on this trait, while the second trait remains under stabilizing selection. The width of the fitness functions that acts on the first and second traits are denoted by $\omega_{1}$ and $\omega_{2}$, respectively.

The maximum number $K$ of reproducing adults may be called the carrying capacity. The $N_{t}(\leq K)$ adults in generation $t$ produce $b N_{t}$ offspring, an expected $R_{t} N_{t}$ of which will survive viability selection. In this way, demographic stochasticity is induced. The sex ratio among parents is always $1: 1$ and $N / 2$ breeding pairs are formed, each of which produces exactly $2 b$ offspring. If the actual number of surviving offspring is larger than $K$, then $K$ individuals are chosen randomly to constitute the next generation of parents. Otherwise, all the surviving offspring serve as parents for the next generation. The effective population size is $N_{e}=4 N /\left(V_{f}+2\right)$, where the variance in family size is $V_{f}=2(1-1 / b)[1-(2 b-1) /(b N-1)]$.

For each parameter combination, a certain number of replicate runs with stochastically independent initial populations were carried out. Each run was over $10^{5}$ generations, unless population extinction had occurred previously. The initial populations were obtained from a preceding initial phase of several hundreds or thousands of generations (depending on $N$ ) during which mutationselection balance had been reached. The number of replicate runs per parameter combination was chosen such that the standard errors were less than $5 \%$ (and often on the order of $1 \%$ ). 
The above theory and several of the consequences derived below are not based on a detailed genetic model, but assume a Gaussian distribution of phenotypes. In particular, the theory does not specify the mechanism by which genetic variation is generated and maintained. Therefore, computer simulations have been performed that use an explicit genetic model and enable the analytic approximations to be tested and the consequences of various assumptions about genetic parameters to be explored. This requires the mechanism by which genetic variability is maintained to be specified. It is assumed that this mechanism is mutation (see Box 3).

\section{Adaptation and Extinction in Changing Environments}

Classic quantitative genetics predicts that a population which experiences selection according to Equation (1) responds by shifting its mean phenotype according to Equation (4). If the optimum phenotype changes continuously, the mean phenotype will lag behind the fitness optimum. If this deviation is too large, the mean fitness may decrease to such an extent that the population cannot replace itself and declines, possibly to extinction. We are primarily concerned here with the role of genetic variation for the extinction risk that results from various forms of temporally varying environments.

\section{Sustained directional change}

For a model in which the optimum moves at a constant rate $\kappa$ per generation, as in Equation (2a), a critical rate of environmental change $\kappa_{c}$ has been identified beyond which extinction is certain because the lag increases from generation to generation, thus decreasing the mean fitness of the population below $\bar{W}<1 / b$, the level at which the population starts to decline. With a smaller population size, genetic drift reduces the genetic variance, which leads to an even larger lag, a further decrease of mean fitness, and rapid extinction (Lynch and Lande 1993; Bürger and Lynch 1995, 1997).

If the rate of environmental change is sufficiently low, then the mean phenotype lags behind the optimum, but after several generations evolves parallel with it. From Equation (4) it can be derived easily that the asymptotic average lag is given by $\kappa / s$, with $s \approx s_{t}$ as in Equation (3) and $V_{G, t}=V_{G \text {,move }}$ the asymptotic genetic variance (Lynch et al. 1991; Lynch and Lande 1993). The genetic load induced by this lag has been called the evolutionary load (Lande and Shannon 1996; see Chapter 9 in Ferrière et al. 2004 for genetic loads) and can be calculated. Indeed, from Equation (5) and the fact that the lag converges to $\kappa / s$, the asymptotic mean fitness is readily calculated to be

$$
\mathbb{E}\left(\bar{W}_{\text {move }}\right) \approx \frac{\omega}{v} \exp \left(-\frac{1}{2} \kappa^{2} /\left(s^{2} v^{2}\right)\right)
$$

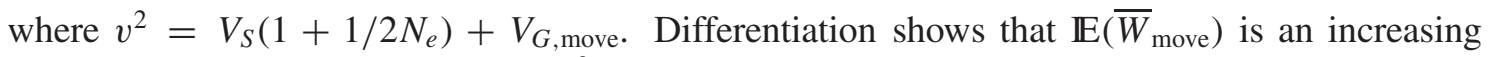
function of the genetic variance if $V_{G \text {,move }}^{3}>2 V_{S}^{2} \kappa^{2}$, that is unless the genetic variance is very large or $\kappa$ is very small (Figure 1a; see Charlesworth 1993b; Lande and Shannon 1996). This, however, does not imply that in a real genetic system the variance evolves such as to maximize mean fitness (see below).

The critical rate of environmental change $\kappa_{c}$ is defined as the value of $\kappa$ at which the population can just replace itself, so that $b \mathbb{E}\left(\bar{W}_{\text {move }}\right)=1$. Unless the population size is very small (less than, say, two dozen) or the stabilizing selection component is extremely weak, $\kappa_{c}$ can be approximated by

$$
\kappa_{c} \approx V_{G, \operatorname{move}} \sqrt{2(\ln b) / V_{S}}
$$




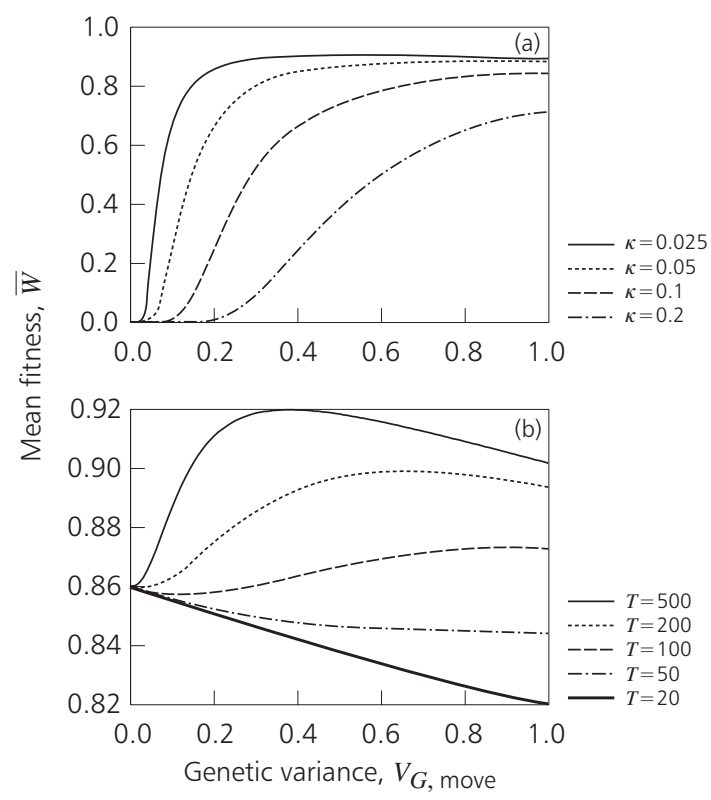

Figure 1 Dependence of mean fitness on genetic variance. (a) displays the mean fitness of a population subject to environmental change according to the moving optimum model, Equations (1) and (2a), as function

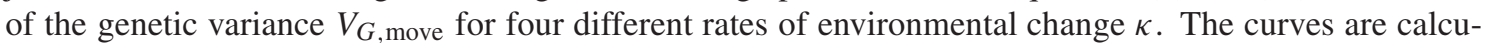
lated from Equation (5). The population is assumed to be infinitely large and the width of the phenotypic fitness function is $\omega=3\left(V_{S}=10\right)$. For large $V_{G \text {, move }}$ the mean fitness decreases because of the stabilizing component of selection. (b) Analagous to (a), but for a periodically changing environment according to Equations (1) and (2b). The amplitude is $A=2 \omega$ and the curves, calculated from Equation (11), are for five different periods, as indicated. Obviously, more genetic variance is beneficial only for long periods $T$. At $V_{G \text {,move }}=0$ the derivative of mean fitness is always negative.

see Lynch and Lande (1993) and Bürger and Lynch (1995). Equations (7) and (8) are deceptively simple because the determinants of the genetic variance have not yet been elucidated. However, it is obvious from Equation (8) that the genetic variance is the major limiting factor for the rate of environmental change that can be tolerated by a population.

This theory can be extended to derive an approximate expression for the mean time to extinction by recognizing that for $\kappa>\kappa_{c}$, the extinction process consists of two phases (Bürger and Lynch 1995). During phase 1, the multiplicative growth rate $R_{t}$ [Equation (6)] decreases to 1 , but the population size remains at the carrying capacity. The length $t_{1}$ of this phase is easily estimated by substituting Equation (7) into Equation (6) and solving the equation $R_{t}=1$ for $t$. This produces

$$
t_{1} \approx-\frac{1}{s} \ln \left(1-\frac{\kappa}{\kappa_{c}}\right) \text {. }
$$

The length $t_{2}$ of the second phase can be obtained by numerical iteration of the recursion $N_{t+1}=$ $R_{t} N_{t}$ until the population size reaches 1 . The second phase is typically much shorter than the first one. This theory for the mean time to extinction, $t_{e}=t_{1}+t_{2}$, produces good approximations if $\kappa \ll \kappa_{c}$ (Figure 2a), although it neglects several sources of stochasticity (fluctuations of $R_{t}$ about its mean, demographic stochasticity, stochasticity and autocorrelation resulting from genetic events like mutation and recombination). Most importantly, it requires knowledge of the actual genetic variance of the population.

An important observation is that the genetic variance actually increases in response to the moving optimum, but only if the population size is sufficiently large (Figure 2b). Therefore, if the population size is higher than a few hundred individuals, the mean time to extinction is longer than predicted by the above theory if this uses the initial genetic variance (see the case $K=512$ 

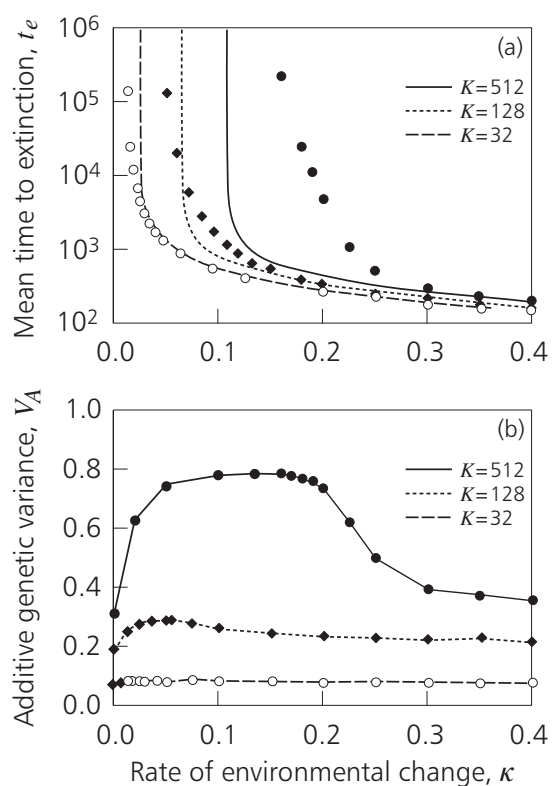

Figure 2 Evolution and extinction in a directionally changing environment. (a) The mean time to extinction as a function of the rate of environmental change $\kappa$ for three different population sizes $K$. Data points are from Monte Carlo simulation, whereas lines are based on the quasi-deterministic theory for $t_{e}$, as described in Equation (9) and the text below. This approximation assumes that the genetic variance does not change after the onset of environmental change, an assumption that is valid only in small populations. Since all genetic variances are less than 1 (see Figure $2 b$ ) and the environmental variance is normalized to 1 , all phenotypic variances are between 1 and 2 . Hence, the value $\kappa=0.1$ corresponds to less than $10 \%$ of a phenotypic standard deviation. (b) The observed genetic variance in the simulations of Figure $2 \mathrm{a}$. The mutational parameters are as in Box 3, and the other parameters are $b=2$ and $\omega=3$. Most data points at which extinction occurred are averages over 100 replicate runs. Source: Numerical data mostly from Bürger and Lynch (1995).

in Figure 2a). Recently, the determinants of this increase were investigated in some detail (Bürger 1999). It was shown that the genetic variance of a quantitative trait determined by many freely recombining loci in a population that, initially, is in mutation-selection-drift equilibrium, increases at least by the factor

$$
\frac{\gamma}{2 \sqrt{u V_{S}}} \frac{N_{e}+V_{S} / \gamma^{2}}{N_{e}+\frac{1}{2} \sqrt{V_{S} /\left(u \gamma^{2}\right)}},
$$

unless $\kappa$ is very small. For increasing $N_{e}$, this converges to $\gamma /\left(2 \sqrt{u V_{S}}\right)$. It was also shown that in sexually reproducing populations in which the trait is controlled by completely linked loci, an increase of variance either does not occur or is much smaller than in freely recombining populations. In asexually reproducing populations, this increase of variance is absent, unless the genomic mutation rate for the trait, and thus the initial equilibrium variance, is extremely small. This flexibility of the genome confers a substantial advantage to sex and recombination if the population is subject to sustained and directional environmental change. Equation (10) also shows that the increase of variance is constrained by the genetic system and not (directly) guided by an optimum principle.

The assumption that environmental change affects only one trait is a gross simplification. In the following we briefly discuss the consequences of pleiotropy. 


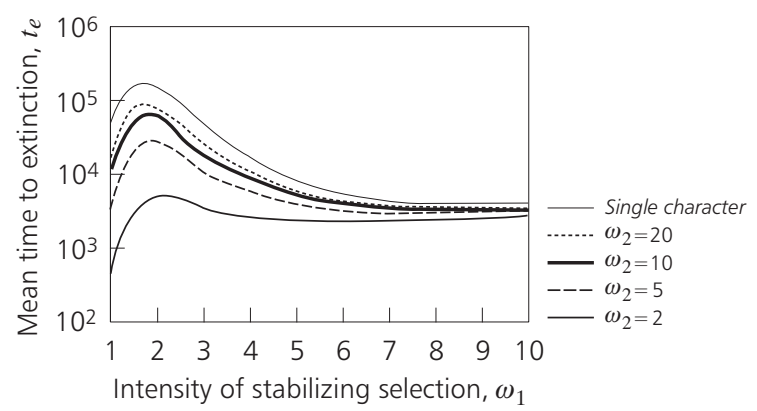

Figure 3 Dependence of mean time to extinction in a directionally changing environment with pleiotropic gene action. Mean extinction time is displayed as a function of stabilizing selection strength $\omega_{1}$ on a character with a moving optimum for various intensities of stabilizing selection $\omega_{2}$ on a second, pleiotropically coupled character with a constant optimum. The rate of environmental change in the direction of the first trait is $\kappa=0.055$ per generation, and for both characters the mutational parameters are as in Box 3. Parameters $\omega_{1}$ and $\omega_{2}$ are the widths of the phenotypic fitness function for each of the two characters. The case of single character (no pleiotropy) is equivalent to $\omega_{2}=\infty$. Other parameters: $K=64, b=2$.

\section{Pleiotropy and changing optima}

Many genes have effects on several characters, and thereby cause a statistical association of heritable variation among different phenotypic traits. This pleiotropic connection of characters may have important consequences for their responses to selection. We employed a simplified version of a model of Lande (1980b), in which two traits are determined by the same set of loci, as described in Box 3. Mutation modifies an allele's contribution to the two characters independently, and there is no selectional correlation between the characters. Figure 3 displays the results of Monte Carlo simulations that evaluate the role of pleiotropy on the evolutionary capacity of the population, in which the first trait is under sustained directional selection while the second is under stabilizing selection, as described in Box 3. The curves in the figure correspond to different strengths of stabilizing selection on the second trait. The top curve represents the case of a single trait, subject to the moving optimum model described in the previous section (Sustained directional change), since this is equivalent to a neutral second trait $\left(\omega_{2}=\infty\right)$.

Figure 3 shows that in the single-character case the dependence of the mean time to extinction on the strength of stabilizing selection is bell shaped and has a maximum near $\omega_{1}=2$, which corresponds to strong selection. This is so because very strong selection focuses the population mean close to the actual optimum, but destroys most of the genetic variance needed to respond to further changes, whereas weak stabilizing selection admits more genetic variance, but leads to a very large lag (see Huey and Kingsolver 1993; Bürger and Lynch 1995). This bell shape of the curve persists if the second character is exposed to increasingly strong selection. However, increasing stabilizing selection on the second character always accelerates population extinction, for three reasons:

- Stabilizing selection on a genetically variable second trait reduces the mean fitness of the population by introducing an additional load.

- As a result of pleiotropy, stabilizing selection on the second character reduces the equilibrium variance of the first one (Lande 1980b; Turelli 1985; Wagner 1989). Therefore, when the optimum starts to move (remember that we allow the population to reach mutationselection equilibrium in a constant environment before environmental change commences), the selection response is reduced; see Equations (c) and (d) in Box 2, and Equation (4).

- A moving optimum increases genetic variance by favoring mutations with positive effects. Since these mutations also affect the second trait, stabilizing selection tries to eliminate them. 
In the present simulations, no correlation between the mutational effects on the two characters was assumed. Therefore, on average, the traits are uncorrelated. Hence, the lag of the wandering character is affected only by the decrease of variation caused by selection on the pleiotropically connected trait. With correlation, however, mutations in a favorable direction for the moving trait have a tendency to push the mean value of the second character away from its optimum. Therefore, selection on the second, now correlated, trait impedes adaptation of the first trait even more, and thereby increases the lag and extinction risk even further (results not shown).

\section{Periodic change}

In a periodically varying environment, Equation (2b), more genetic variance is not necessarily beneficial for population persistence. This can be seen from Figure 1b, which is based on the following approximation for the mean fitness averaged over one full cycle, after a sufficiently long initial phase has elapsed,

$$
\bar{W}_{\text {per }} \approx \frac{\omega}{v} \exp \left(-\lambda+\frac{1}{4} \lambda^{2}\right),
$$

where the expected log mean fitness (Lande and Shannon 1996; Bürger 1999) is

$$
\lambda=\frac{1}{2} \mathbb{E}\left(\Delta^{2}\right) /\left(V_{S}+V_{G}\right) \approx \frac{A^{2} \pi^{2}}{V_{S}\left(s^{2} T^{2}+4 \pi^{2}\right)} .
$$

Here, $s$ is given by Equation (3) with $V_{G, t}$ equal to the genetic variance averaged over a full cycle. The above equations assume a large population size but, as shown by comparison with Monte Carlo simulations, yield close approximations for populations above 100-200 individuals.

The detailed dynamics of evolution and extinction for both finite sexual and asexual populations were investigated by Monte Carlo simulations, as described in Box 3. No assumptions are imposed on the distribution of phenotypic (or genotypic) values. Some of the results are summarized in Figure 4. Further results are found in Bürger (1999, 2000). Figure 4 displays the mean time to extinction and the average genetic variance of a freely recombining sexual population, of a nonrecombining sexual population, and of an asexual population as a function of $\kappa=4 A / T$. These populations differ substantially in their initial genetic variance (with recombination and sex more genetic variance is maintained at mutation-selection balance than without; see Bürger 1999), as well as in their ability to adapt their genetic variance to a higher, selectively favorable, level (Figure 4b). Figure 4a shows that for intermediate periods of $T$, the freely recombining population persists for much longer than the two other populations. The reason is that in this case the populations have to adapt to the changing optimum, which requires much genetic variance. The freely recombining population not only has a higher initial variance, but is also able to increase its level of variance, and thus obtains a substantial advantage over the two other populations. For very long periods, all three types of population harbor enough genetic variance to track the optimum. If the optimum changes rapidly, so that it returns to its initial state every few generations, more genetic variance is not beneficial. In this case, it makes sense for a population to stay where it is and wait until the environmental optimum returns. Clearly, this requires that the population be able to maintain a minimum viable population size during periods of low fitness.

\section{Stochastic fluctuations}

Several types of models have been investigated to evaluate population persistence in stochastically fluctuating environments. A large body of literature on environmental stochasticity neglects the genetic structure of populations and studies extinction of monomorphic populations under a variety of assumptions about the demography and ecology of the population [see Chapter 2 in Ferrière et al. 2004, and Bürger and Lynch (1997) for reviews]. 


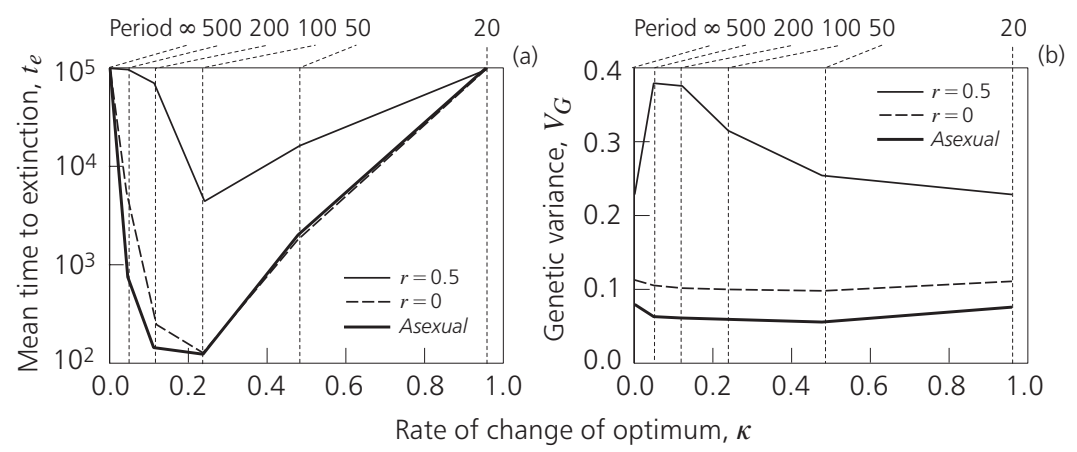

Figure 4 Evolution and extinction in a periodic environment. (a) The mean time to extinction as a function of $\kappa=4 A / T$ for a freely recombining sexual population, a nonrecombining sexual population, and a diploid asexual population. Here, $\kappa=4 A / T$ can be interpreted as the rate of change of the optimum, averaged over one full cycle (during which the optimum moves $4 A$ units, measured in multiples of $V_{E}$ ). Dynamically, an infinitely long period $T$ is equivalent to a resting optimum. The amplitude $A$ of the periodic optimum is chosen to be $2 \omega$, which implies that at the most extreme position of the optimum ( $A$ units from the origin) the originally optimal phenotype (at position 0 ) has a fitness of $13.5 \%$. Data points are from Monte Carlo simulation. Parameters: $K=256, b=5, \omega=3$, and the number of loci and the mutation parameters are as in Box 3. (b) The observed genetic variance in the simulations of Figure 4a.

Recently, more attention has been paid to the role of genetic variability in population survival in randomly varying environments. Charlesworth (1993b) and Lande and Shannon (1996) investigated fluctuating stabilizing selection on a quantitative trait by assuming that the optimum $\hat{P}_{t}$ in Equation (1) follows a linear stationary Markov process with mean zero, variance $V_{\theta}$, and autocorrelation $c$ between -1 and 1. They assumed the Gaussian phenotypic model of Box 2 and a constant genetic variance. Charlesworth (1993b) showed that the expected log mean fitness increases with increased genetic variance if

$$
\frac{V_{\theta}}{V_{S}}>\frac{2(1-c)}{1+c} \text {. }
$$

Thus, genetic variation is only beneficial if the variance of the fluctuations is high or if the process is highly autocorrelated, in which case adaptation, that is tracing the optimum, increases the mean fitness. For a continuous-time model (which precludes large instantaneous fluctuations), Lande and Shannon (1996) also showed that in autocorrelated environments more genetic variation is usually beneficial, but for uncorrelated environments they found that more genetic variation always decreases mean fitness.

These authors did not consider population extinction, but assumed an unlimited reproductive potential and a constant variance. Bürger (1999) employed the simulation model described in Box 3 to study population extinction for the case of no autocorrelation $(c=0)$. He found that, even for $V_{\theta} / V_{S} \approx 1$, higher levels of genetic variation can slightly enhance the mean persistence times. Nevertheless, with such an environmental change a high reproductive rate is much more effective at improving population longevity than a high genetic variance. Bürger and Lynch (1995) considered a mixture of the moving optimum model, Equation (2a), and the above model of environmental stochasticity with $c=0$ by assuming that the optimum $\hat{P}_{t}$ evolves according to $\hat{P}_{t}=\kappa t+\varepsilon_{t}$, where $\varepsilon_{t}$ is a normally distributed random number with a mean of zero and variance of $V_{\theta}$. They showed that, relative to a smoothly moving optimum, superimposition of mild levels of stochasticity can reduce the mean time to extinction by one or more orders of magnitude. This reduction is most pronounced if, without stochastic fluctuations, the expected mean extinction time of the population is above several thousands of generations, so that from environmental change alone the extinction risk is small. This indicates that the synergistic interactions of environmental changes that separately cause only a minor risk can cause rapid extinction. The amount by which 
the critical rate of environmental change is reduced by small random fluctuations of the optimum has been calculated by Lynch and Lande (1993).

\section{Single abrupt change}

Gomulkiewicz and Holt (1995) investigated environmental change caused by a single shift of the optimum phenotype of a quantitative trait to a new constant value. Such an abrupt change reduces the mean fitness of the population, possibly to the extent that the multiplicative growth rate falls below 1. If the maladaptation caused by the shift is too severe, rapid extinction of the population will be the consequence. Survival of a population after such a shift may be possible if adaptive variation admits sufficiently rapid evolution toward the new optimum, thus leading to an increase in growth rate. Even if this is the case, the population size decreases for some time because of the low initial fitness. If it decays below a certain critical value, it may be highly endangered by demographic stochasticity. Therefore, "the problem of population persistence in a novel environment can be viewed as a race between two processes, one demographic, another evolutionary" (Maynard Smith 1998; see also Gomulkiewicz and Holt 1995).

\section{Concluding Comments}

What is the practical value of these models for the conservation biologist? Given the limitations conservation biology faces in practice, it may well be that such models only offer possible explanations for the extinction of populations rather than provide measures to ensure their survival. For small populations of up to a few hundred individuals, extinction is an almost certain event, even under very moderate, but sustained, changes of the environment that shift the optimal value of a trait by just a few percent of a phenotypic standard deviation per generation. If environmental change proceeds too fast (on the order of $10 \%$ of a standard deviation or more), even a large population size cannot guarantee survival for a long period, in particular if additional stochastic fluctuations occur in the environment, or if pleiotropically connected traits are under stabilizing selection (see Figures 2 and 3; and Bürger and Lynch 1995). This is a crucial difference to extinction risks caused by genetic factors, such as the accumulation of deleterious mutants (Chapter 9 in Ferrière et al. 2004), or by demographic and environmental stochasticity (Chapter 2 in Ferrière et al. 2004), in which the risk decreases rapidly with increasing population size. Population size is the parameter that can be influenced most directly by conservation biology efforts; however, as shown above, a large population size is not always sufficient for population survival during prolonged episodes of environmental change. The case might be more promising with populations that face one abrupt shift to a new constant environment. Here, direct measures may be taken to support the population during the period when it is below the critical density.

The consequences of genetic variability for population survival depend on the kind of environmental change. A high level of genetic variance improves population performance under continuous directional change, under a single abrupt shift of the optimum, under a cyclically varying environment (if the amplitude is not too small and the period is long), and under stochastic fluctuations with high variance or a high autocorrelation. In such cases, the only means to survive is adaptation to the environment, which requires genetic variance. In a constant environment, in a periodically changing one with a short period, and in a randomly fluctuating environment with small variance and no autocorrelation, more genetic variance may even be slightly detrimental, because it increases the load caused by stabilizing selection (see Slatkin and Lande 1976; Charlesworth 1993a, 1993b; Lande and Shannon 1996). In such environments, the production of many offspring may substantially increase population persistence (Bürger 1999). 
In our models, several factors with possibly strong influences on population persistence were not considered. Populations were assumed to be panmictic, the environment was unstructured, the life cycle was very simple, rates of mutation were assumed to be constant, and unconditionally deleterious mutations were neglected. For a discussion of complex life cycles see Chapter 7 in Ferrière et al. 2004, for the effects of a metapopulation structure and a spatially structured environment, see Part D. Chapter 8 in Ferrière et al. 2004 discusses mechanisms for variable mutation rates.

Shifts in the population mean of more than several standard deviations have been observed in artificial selection experiments, which shows that at least for some characters there is enough genetic variation to respond to large changes [see Barton and Keightley (2002) for references]. These shifts, however, at least partially resulted from the spread of recessive deleterious alleles. Therefore, when selection was relaxed, the mean often returned to a value between its initial value and its maximum value. In addition, lines were lost in such experiments because of reproductive failure. Whether a particular trait has the potential for substantial evolution depends on a multitude of genetic and demographic details and, in general, is hardly predictable.

\section{References}

Barton NH \& Turelli M (1989). Evolutionary quantitative genetics: How little do we know? Annual Revue of Genetics 23:337-370

Barton NH \& Keightley (2002). Understanding quantitative genetic variation. Nature Reviews, Genetics 3:11-21

Bulmer MG (1980). The Mathematical Theory of Quantitative Genetics. Oxford, UK: Clarendon Press

Bürger R (1999). Evolution of genetic variability and the advantage of sex and recombination in changing environments. Genetics 153:1055-1069

Bürger R (2000). The Mathematical Theory of Selection, Recombination, and Mutation. Chichester, UK: Wiley \& Sons

Bürger R \& Gimelfarb A (2002). Fluctuating environments and the mode of mutation in maintaining genetic variation. Genetical Research 80:31-46

Bürger R \& Lynch M (1995). Evolution and extinction in a changing environment: A quantitativegenetic analysis. Evolution 49:151-163

Bürger R \& Lynch M (1997). Adaptation and extinction in changing environments. In Environmental Stress, Adaptation and Evolution, eds. Bijlsma R \& Loeschcke V, pp. 209-239. Basel, Switzerland: Birkhäuser

Bürger R, Wagner GP \& Stettinger F (1989). How much heritable variation can be maintained in finite populations by mutation-selection balance? Evolution 43:1748-1766

Charlesworth B (1993a). The evolution of sex and recombination in a varying environment. Journal of Heredity 84:345-450

Charlesworth B (1993b). Directional selection and the evolution of sex and recombination. Genetical Research 61:205-224

Charlesworth B \& Charlesworth D (1998). Some evolutionary consequences of deleterious mutations. Genetica 102/103:3-19

Crow JF \& Kimura M (1964). The theory of genetic loads. In Proceedings of the XI International Congress of Genetics, ed. Geerts S, pp. 495-505. Oxford, UK: Pergamon Press

Falconer DS \& Mackay TFC (1996). Introduction to Quantitative Genetics, Fourth Edition. New York, NY, USA: Addison Wesley Longman

Ferrière R, Dieckmann U \& Couvet D (2004). Evolutionary Conservation Biology. Cambridge, UK: Cambridge University Press. 
Fisher R (1918). The correlation between relatives on the supposition of Mendelian inheritance. Transactions of the Royal Society of Edinburgh 52:399-433

Fisher RA (1930). The Genetical Theory of Natural Selection. Oxford, UK: Clarendon Press

Fisher RA (1941). Average excess and average effect of a gene substitution. Annals of Eugenics 11:53-63

Galton F (1889). Natural Inheritance. London, UK: MacMillan

Gomulkiewicz R \& Holt RD (1995). When does evolution by natural selection prevent extinction? Evolution 49:201-207

Huey RB \& Kingsolver JG (1993). Evolution of resistance to high temperature in ectotherms. The American Naturalist 142:S21-S46

Kondrashov AS \& Yampolsky LY (1996a). High genetic variability under the balance between symmetric mutation and fluctuating stabilizing selection. Genetical Research 68:157-164

Kondrashov AS \& Yampolsky LY (1996b). Evolution of amphimixis and recombination under fluctuating selection in one and many traits. Genetical Research 68:165-173

Lande R (1975). The maintenance of genetic variation by mutation in a polygenic character with linked loci. Genetical Research 26:221-235

Lande R (1976). Natural selection and random genetic drift in phenotypic evolution. Evolution 30:314-334

Lande R (1979). Quantitative genetic analysis of multivariate evolution applied to brain: Body size allometry. Evolution 34:402-416

Lande R (1980b). The genetic covariance between characters maintained by pleiotropic mutations. Genetics 94:203-215

Lande R \& Shannon S (1996). The role of genetic variation in adaptation and population persistence in a changing environment. Evolution 50:434-437

Latter BDH (1970). Selection in finite population with multiple alleles. II: Centripetal selection, mutation, and isoallelic variation. Genetics 66:165-186

Lush JL (1937). Animal Breeding Plans. Ames, IA, USA: Iowa State University Press

Lynch M \& Lande R (1993). Evolution and extinction in response to environmental change. In Biotic Interactions and Global Change, eds. Kareiva PM, Kingsolver JG \& Huey RB, pp. 234-250. Sunderland, MA, USA: Sinauer Associates Inc.

Lynch M \& Walsh JB (1998). Genetics and Analysis of Quantitative Traits. Sunderland, MA, USA: Sinauer Associates Inc.

Lynch M, Gabriel W \& Wood AM (1991). Adaptive demographic responses of plankton populations to environmental change. Limnology and Oceanography 36:1301-1312

Maynard Smith J (1998). Evolutionary Genetics, Second Edition. Oxford, UK: Oxford University Press

Mayo O (1987). The Theory of Plant Breeding, Second Edition. Oxford, UK: Clarendon Press

Pearson K (1903). Mathematical contributions to the theory of evolution. XI. On the influence of natural selection on the variability and correlation of organs. Philosophical Transactions of the Royal Society A 200:1-66

Slatkin M \& Lande R (1976). Niche width in a fluctuating environment-density dependent model. The American Naturalist 110:31-55

Smith HF (1936). A discriminant function for plant selection. Annals of Eugenics 7:240-250

Turelli M (1984). Heritable genetic variation via mutation-selection balance: Lerch's zeta meets the abdominal bristle. Theoretical Population Biology 25:138-193

Turelli M (1985). Effects of pleiotropy on predictions concerning mutation-selection balance for polygenic traits. Genetics 111:165-195

Turelli M (1988). Population genetic models for polygenic variation and evolution. In Proceedings of the Second International Conference on Quantitative Genetics, eds. Weir BS, Eisen EJ, 
Goodman NM \& Namkoong G, pp. 601-618. Sunderland, MA, USA: Sinauer Associates Inc.

Turelli M \& Barton NH (1994). Genetic and statistical analyses of strong selection on polygenic traits: What, me normal? Genetics 138:913-941

Wagner GP (1989). Multivariate mutation-selection balance with constrained pleiotropic effects. Genetics 122:223-234

Wright S (1921). Systems of mating. Genetics 6:111-178 